\title{
An Autocorrelation Term Method for Curve Fitting
}

\author{
Louis M. Houston \\ The Louisiana Accelerator Center, The University of Louisiana at Lafayette, Lafayette, LA 70504-4210, USA \\ Correspondence should be addressed to Louis M. Houston; houston@louisiana.edu
}

Received 7 May 2013; Accepted 8 July 2013

Academic Editors: K. Djidjeli, J. Kou, and M. Qatu

Copyright (C) 2013 Louis M. Houston. This is an open access article distributed under the Creative Commons Attribution License, which permits unrestricted use, distribution, and reproduction in any medium, provided the original work is properly cited.

\begin{abstract}
The least-squares method is the most popular method for fitting a polynomial curve to data. It is based on minimizing the total squared error between a polynomial model and the data. In this paper we develop a different approach that exploits the autocorrelation function. In particular, we use the nonzero lag autocorrelation terms to produce a system of quadratic equations that can be solved together with a linear equation derived from summing the data. There is a maximum of $2 M$ solutions when the polynomial is of degree $M$. For the linear case, there are generally two solutions. Each solution is consistent with a total error of zero. Either visual examination or measurement of the total squared error is required to determine which solution fits the data. A comparison between the comparable autocorrelation term solution and linear least squares shows negligible difference.
\end{abstract}

\section{Introduction}

Curve fitting is the process of fitting a curve, in this case a polynomial, to a set of data points. There are different types of curve fitting, but we will discuss only the most popular method, the method of least squares [1].

It is assumed that the data consists of fluctuations about an ideal curve. These fluctuations create an error between the polynomial model and the actual data. After computing a total squared error, we can apply calculus to minimize the squared error with respect to the coefficients of the polynomial. This produces a set of linear equations called the normal equations. The coefficients are derived from solving the normal equations [2].

A different approach to the problem is as follows.

We propose that the data we are considering consists of a deterministic component and a random component with zero mean. The random component does not correlate with the deterministic component and it does not correlate with itself at nonzero lag. We want to extract only the portion of the data that is correlative at nonzero lag. This requires the computation of nonzero lag autocorrelation terms and produces a system of quadratic equations [3]. After substitution based on a linear equation derived from summing the data, the system of quadratic equations consists of $M$ equations with $M$ unknowns, where $M$ is the degree of the polynomial. This results in a maximum of $2 M$ solutions. Each solution is consistent with a total error between the polynomial and the data that is equal to zero. Either visual examination or measurement of the total squared error is required to determine which solution fits the data.

In this paper, we derive the autocorrelation term method and compare it to linear least squares.

\section{The Autocorrelation Term Method}

Consider a set of data $\left(x_{i}, y_{i}\right)(i=1,2,3, \ldots, N)$. Characterize the $y$ data as the sum of a polynomial function $y\left(x_{i}\right)$ and a discrete, zero-mean random variable [4] $Z_{i}$ :

$$
\begin{gathered}
Z_{i}: \Omega \rightarrow \mathbb{R}, \\
y_{i}=y\left(x_{i}\right)+Z_{i} .
\end{gathered}
$$

Consequently, we can write

$$
\begin{aligned}
& y_{i+m} y_{i}=\left(y\left(x_{i+m}\right)+Z_{i+m}\right)\left(y\left(x_{i}\right)+Z_{i}\right), \\
&= y\left(x_{i+m}\right) y\left(x_{i}\right)+Z_{i+m} y\left(x_{i}\right)+Z_{i} y\left(x_{i+m}\right)+Z_{i+m} Z_{i}, \\
& m \in\{1,2, \ldots\} .
\end{aligned}
$$

Let $N \gg m$ 
Summation yields

$$
\sum_{i=1}^{N-m} y_{i+m} y_{i}=\sum_{i=1}^{N-m} y\left(x_{i+m}\right) y\left(x_{i}\right)
$$

since the cross-correlation [5] and autocorrelation terms with $Z_{i}$ must be zero. The polynomial function of degree $M$ can be written as

$$
\begin{aligned}
y\left(x_{i}\right) & =a_{0}+a_{1} x_{i}+a_{2} x_{i}^{2}+\cdots+a_{M} x_{i}^{M} \\
& =\sum_{j=0}^{M} a_{j} x_{i}^{j} .
\end{aligned}
$$

Therefore, (3) becomes the system of equations:

$$
\begin{array}{r}
\sum_{i=1}^{N-m} y_{i+m} y_{i}=\sum_{i=1}^{N-m}\left(\sum_{j=0}^{M} a_{j} x_{i+m}^{j}\right)\left(\sum_{j=0}^{M} a_{j} x_{i}^{j}\right), \\
m=1,2, \ldots, M .
\end{array}
$$

Using (1), we can write the average

$$
\begin{aligned}
\left\langle y_{i}\right\rangle & =\left\langle y\left(x_{i}\right)+Z_{i}\right\rangle \\
& =\left\langle y\left(x_{i}\right)\right\rangle+\left\langle Z_{i}\right\rangle .
\end{aligned}
$$

We know that $\left\langle Z_{i}\right\rangle=0$, so

$$
\left\langle y_{i}\right\rangle=\left\langle y\left(x_{i}\right)\right\rangle
$$

which implies

$$
\sum y_{i}=\sum y\left(x_{i}\right)
$$

or

$$
\sum_{i=1}^{N} y_{i}=\sum_{i=1}^{N} \sum_{j=0}^{M} a_{j} x_{i}^{j} .
$$

There are a maximum of $2 M$ solutions to the system of equations created by (5) and (9) for the coefficients $\left\{a_{0}, a_{1}, \ldots, a_{M}\right\}$. Observe that the total error, $E$, is presumed to be zero:

$$
E=\sum_{i=1}^{N}\left(\sum_{j=0}^{M} a_{j} x_{i}^{j}-y_{i}\right)=0 .
$$

\section{The Least-Squares Straight Line}

Before examining the autocorrelation method, it is worth becoming familiar with the special case of fitting a straight line in the least-squares sense. We fit

$$
y(x)=a_{0}+a_{1} x
$$

to a set of data $\left(x_{i}, y_{i}\right)(i=1,2,3, \ldots, N)$. We have two parameters $a_{0}$ and $a_{1}$ and want to minimize the function of two variables $\left(a_{0}\right.$ and $\left.a_{1}\right)$ :

$$
F\left(a_{0}, a_{1}\right)=\sum_{i=1}^{N}\left[y\left(x_{i}\right)-y_{i}\right]^{2} .
$$

This becomes

$$
F\left(a_{0}, a_{1}\right)=\sum_{i=1}^{N}\left[a_{0}+a_{1} x_{i}-y_{i}\right]^{2} .
$$

Apply the calculus method

$$
\begin{gathered}
\frac{\partial F\left(a_{0}, a_{1}\right)}{\partial a_{0}}=2 \sum\left(a_{0}+a_{1} x_{i}-y_{i}\right)=0, \\
\frac{\partial F\left(a_{0}, a_{1}\right)}{\partial a_{1}}=2 \sum\left(a_{0}+a_{1} x_{i}-y_{i}\right) x_{i}=0 .
\end{gathered}
$$

Dropping factor 2 and rewriting, we get

$$
\begin{gathered}
a_{0} N+a_{1} \sum x_{i}=\sum y_{i}, \\
a_{0} \sum x_{i}+a_{1} \sum x_{i}^{2}=\sum x_{i} y_{i} .
\end{gathered}
$$

Solving these equations for $a_{0}$ and $a_{1}$ yields

$$
\begin{gathered}
a_{0}=\frac{1}{N} \sum y_{i}-\frac{1}{N} \sum x_{i}\left(\frac{\sum x_{i} y_{i}-(1 / N) \sum y_{i} \sum x_{i}}{\sum x_{i}^{2}-\left(\left(\sum x_{i}\right)^{2} / N\right)}\right), \\
a_{1}=\frac{\sum x_{i} y_{i}-(1 / N) \sum y_{i} \sum x_{i}}{\sum x_{i}^{2}-\left(\left(\sum x_{i}\right)^{2} / N\right)} .
\end{gathered}
$$

\section{The Autocorrelation Term Method for $M=1$}

For $M=1$, system (5) becomes

$$
\begin{aligned}
\sum_{i=1}^{N-1} y_{i+1} y_{i}= & \sum_{i=1}^{N-1}\left(a_{0}+a_{1} x_{i+1}\right)\left(a_{0}+a_{1} x_{i}\right) \\
= & (N-1) a_{0}^{2}+\left(\sum_{i=1}^{N-1}\left(x_{i+1}+x_{i}\right)\right) a_{0} a_{1} \\
& +\left(\sum_{i=1}^{N-1} x_{i+1} x_{i}\right) a_{1}^{2} .
\end{aligned}
$$

Suppressing the summation limits, we can write

$$
\begin{aligned}
\sum y_{i+1} y_{i}= & (N-1) a_{0}^{2}+\left(\sum\left(x_{i+1}+x_{i}\right)\right) a_{0} a_{1} \\
& +\left(\sum x_{i+1} x_{i}\right) a_{1}^{2} .
\end{aligned}
$$

Equation (9) becomes

$$
\sum y_{i}=N a_{0}+a_{1} \sum x_{i} .
$$

Solving (19) for $a_{0}$ yields

$$
a_{0}=\frac{1}{N}\left(\sum y_{i}-a_{1} \sum x_{i}\right) .
$$


Squaring yields

$$
a_{0}^{2}=\frac{1}{N^{2}}\left(\left(\sum y_{i}\right)^{2}-2 a_{1} \sum y_{i} \sum x_{i}+a_{1}^{2}\left(\sum x_{i}\right)^{2}\right)
$$

Now substitute (20) and (21) into (18) and organize the terms:

$$
\begin{aligned}
& {\left[\frac{(N-1)}{N^{2}}\left(\sum x_{i}\right)^{2}+\left(\sum x_{i+1} x_{i}\right)\right.} \\
& \left.-\frac{1}{N} \sum\left(x_{i+1}+x_{i}\right)\left(\sum x_{i}\right)\right] a_{1}^{2} \\
& \quad+\left[\frac{1}{N} \sum\left(x_{i+1}+x_{i}\right)\left(\sum y_{i}\right)-\frac{2(N-1)}{N^{2}} \sum y_{i} \sum x_{i}\right] \\
& \quad \times a_{1}+\frac{(N-1)}{N^{2}}\left(\sum y_{i}\right)^{2}-\sum y_{i+1} y_{i}=0 .
\end{aligned}
$$

This is in the form of a quadratic equation that we can write as

$$
A a_{1}^{2}+B a_{1}+C=0
$$

where, with reference to (22), $A$ is the precursor of $a_{1}^{2}, B$ is the precursor of $a_{1}$, and $C$ is the residual expression.

The solutions are the roots

$$
a_{1}=\frac{-B \pm \sqrt{B^{2}-4 A C}}{2 A} .
$$

$a_{0}$ is found using (20).

\section{The Autocorrelation Term Method for Higher Values of $M$}

The autocorrelation term method for curve fitting with an $M$ th degree polynomial requires the solution of one linear equation and $M$ quadratic equations with $M+1$ variables which reduces to $M$ quadratic equations with $M$ variables. This can produce $2 M$ solutions [6]. For example, consider the $M=2$ case. System (5) can be written as

$$
\begin{aligned}
& \sum y_{i+1} y_{i}=\sum\left(a_{0}+a_{1} x_{i+1}+a_{2} x_{i+1}^{2}\right)\left(a_{0}+a_{1} x_{i}+a_{2} x_{i}^{2}\right), \\
& \sum y_{i+2} y_{i}=\sum\left(a_{0}+a_{1} x_{i+2}+a_{2} x_{i+2}^{2}\right)\left(a_{0}+a_{1} x_{i}+a_{2} x_{i}^{2}\right) .
\end{aligned}
$$

Equation (9) can be written as

$$
\sum y_{i}=\sum\left(a_{0}+a_{1} x_{i}+a_{2} x_{i}^{2}\right)
$$

From (26) we can write

$$
a_{0}=\frac{1}{N} \sum y_{i}-\frac{a_{1}}{N} \sum x_{i}-\frac{a_{2}}{N} \sum x_{i}^{2} .
$$

Substitute (27) into (25):

$$
\begin{aligned}
\sum y_{i+1} y_{i}=\sum( & \frac{1}{N} \sum y_{i}+\left(x_{i+1}-\frac{1}{N} \sum x_{i}\right) a_{1} \\
& \left.+\left(x_{i+1}^{2}-\frac{1}{N} \sum x_{i}^{2}\right) a_{2}\right) \\
& *\left(\frac{1}{N} \sum y_{i}+\left(x_{i}-\frac{1}{N} \sum x_{i}\right) a_{1}\right. \\
& \left.+\left(x_{i}^{2}-\frac{1}{N} \sum x_{i}^{2}\right) a_{2}\right), \\
\sum y_{i+2} y_{i}=\sum & \frac{1}{N} \sum y_{i}+\left(x_{i+2}-\frac{1}{N} \sum x_{i}\right) a_{1} \\
& \left.+\left(x_{i+2}^{2}-\frac{1}{N} \sum x_{i}^{2}\right) a_{2}\right) \\
* & \frac{1}{N} \sum y_{i}+\left(x_{i}-\frac{1}{N} \sum x_{i}\right) a_{1} \\
& \left.+\left(x_{i}^{2}-\frac{1}{N} \sum x_{i}^{2}\right) a_{2}\right) .
\end{aligned}
$$

Equations (28) are two quadratic equations in $a_{1}$ and $a_{2}$. This can produce four solutions. In general, these types of systems can be solved on a Grobner basis using Buchberger's algorithm [7].

\section{A Comparison of the Autocorrelation Term Method to Linear Least Squares}

We have constructed synthetic data consisting of the sum of a line and a random variable. The line has an intercept of fifteen and a slope of five. The random variable is Gaussian with a zero mean. The amplitude of the random variable is twenty. There are a total of one hundred equally spaced data points. Figure 1 shows the data, the least squares fit, and the autocorrelation term fit for $a_{1}=\left(-B+\sqrt{B^{2}-4 A C}\right) / 2 A$. Figure 2 shows the data, the least squares fit, and the autocorrelation term fit for $a_{1}=\left(-B-\sqrt{B^{2}-4 A C}\right) / 2 A$. In this example, we find that the quadratic roots are negatives of one another. This occurs because $x_{i}=i-1$ which implies that $B=0$. This follows from (22) which for $B=0$ implies

$$
\sum\left(x_{i+1}+x_{i}\right)=\frac{2(N-1)}{N} \sum x_{i} .
$$

This becomes

$$
\sum(2 i-1)=\frac{2(N-1)}{N} \sum(i-1)
$$

which reduces to

$$
\sum_{i=1}^{N-1}(2 i-1)=(N-1)^{2} .
$$

\section{Conclusions}

We have derived a method for computing polynomial curve fits to data based on terms from the autocorrelation function. The method produces $M$ quadratic equations with $M$ 


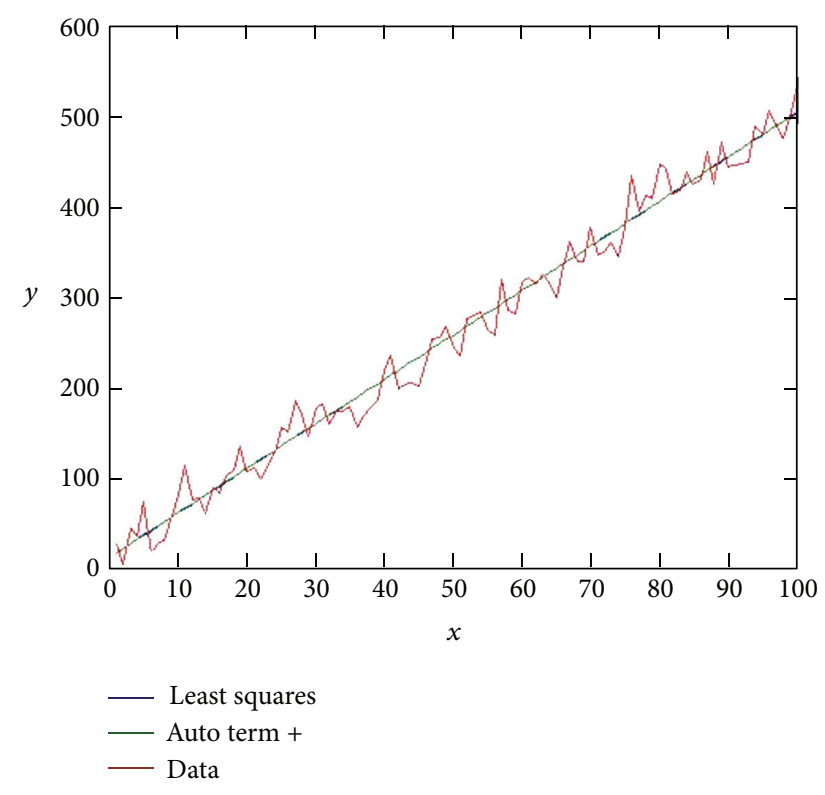

FIGURE 1: An overlay of the data, the least-squares fit, and the autocorrelation term fit for $a_{1}=\left(-B+\sqrt{B^{2}-4 A C}\right) / 2 A$.

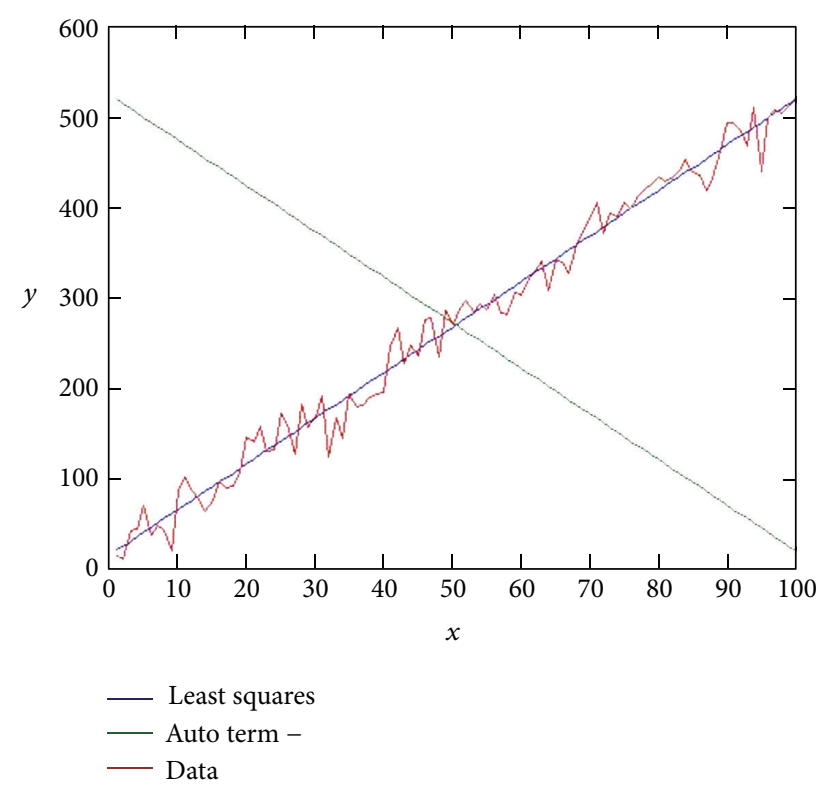

FIGURE 2: An overlay of the data, the least-squares fit, and the autocorrelation term fit for $a_{1}=\left(-B-\sqrt{B^{2}-4 A C}\right) / 2 A$.

variables for a polynomial of degree $M$. The solutions of this system maximally produce $2 M$ curves. Each solution is consistent with a total error between the polynomial and the data that is equal to zero. The proper curve can be selected with either visual examination or measurement of the total squared error. We tested this method with a linear curve fit and compared it to linear least squares. There is negligible difference between the comparable solutions.

\section{Acknowledgment}

Discussions with Gwen Houston and Dominique Lueckenhoff are greatly appreciated.

\section{References}

[1] S. D. Christian, E. H. Lane, and F. Garland, "Linear least-squares analysis: a caveat and a solution," Journal of Chemical Education, vol. 51, no. 7, p. 475, 1974.

[2] R. J. O'Dowd, “The Wiener-Levinson algorithm and ill-conditioned normal equations," Geophysical Journal International, vol. 106, no. 2, pp. 399-406, 1991.

[3] Y. Kinoshita, T. Asakura, and M. Suzuki, "Autocorrelation of Gaussian-Beam fluctuation caused by a random medium," Journal of the Optical Society of America, vol. 58, no. 8, pp. 1040-1047, 1968.

[4] K. Wong and S. Redman, "The recovery of a random variable from a noisy record with application to the study of fluctuations in synaptic potentials," Journal of Neuroscience Methods, vol. 2, no. 4, pp. 389-409, 1980.

[5] M. E. Keating, F. Bonnier, and H. J. Byrne, "Spectral crosscorrelation as a supervised approach for the analysis of complex Raman datasets: the case of nanoparticles in biological cells," Analyst, vol. 137, pp. 5792-5802, 2012.

[6] A. Denis, "A discussion of the cases when two quadratic equations involving two variables can be solved by the method of quadratics," The American Mathematical Monthly, vol. 10, no. 89, pp. 192-199, 1903.

[7] B. Buchberger, "An algorithm for finding the basis elements of the residue class ring of a zero dimensional polynomial ideal," Journal of Symbolic Computation, vol. 41, no. 3-4, pp. 475-511, 2006. 


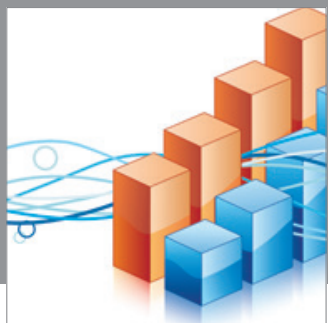

Advances in

Operations Research

mansans



The Scientific World Journal
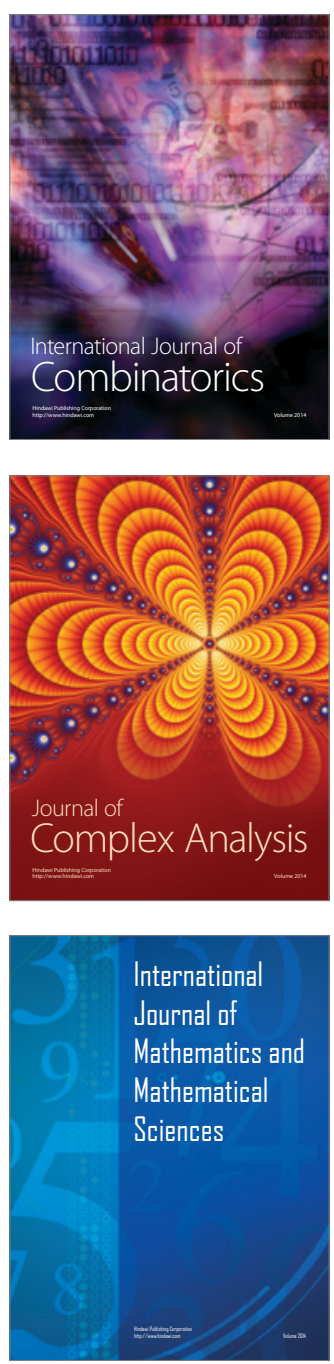
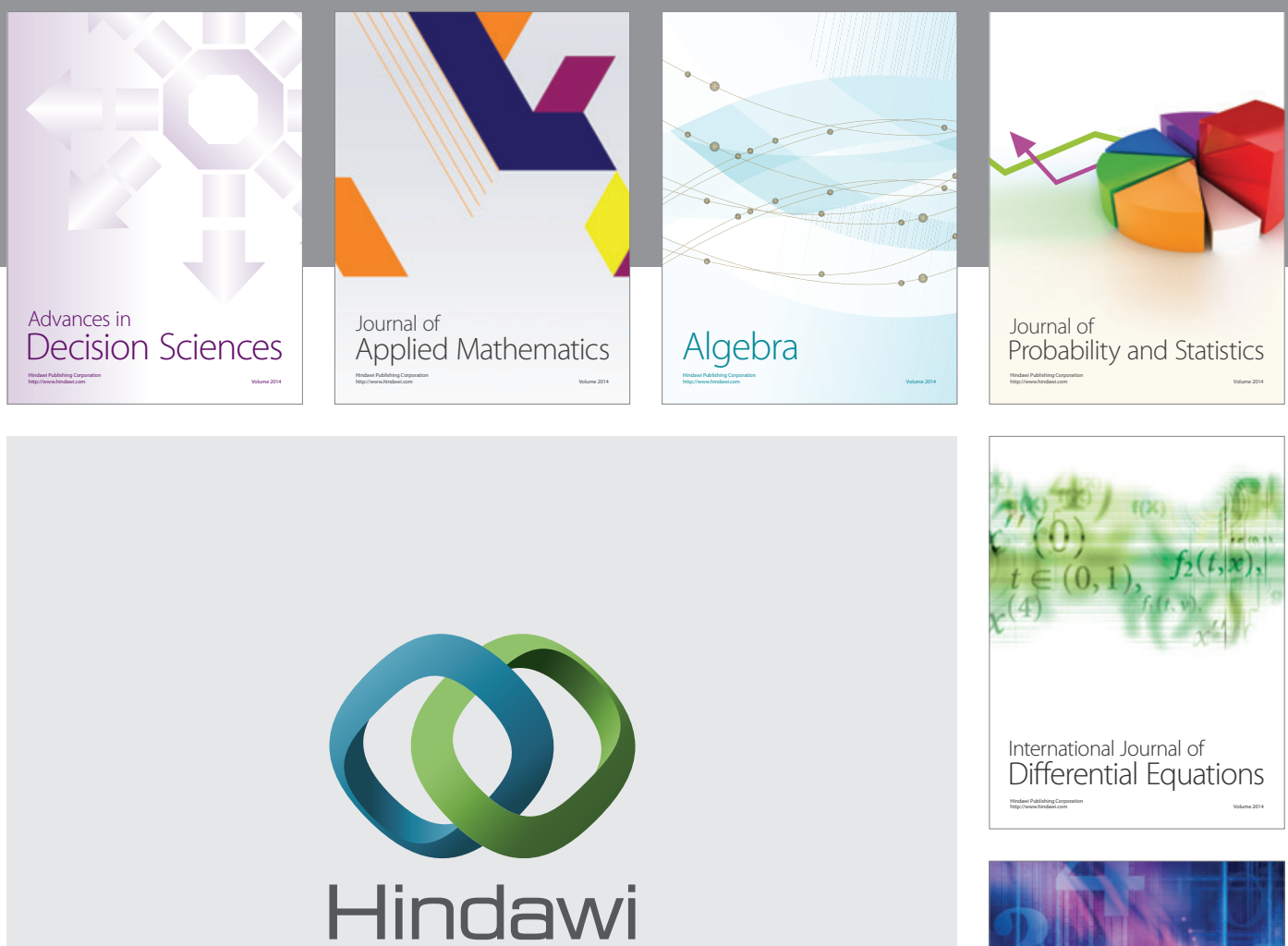

Submit your manuscripts at http://www.hindawi.com
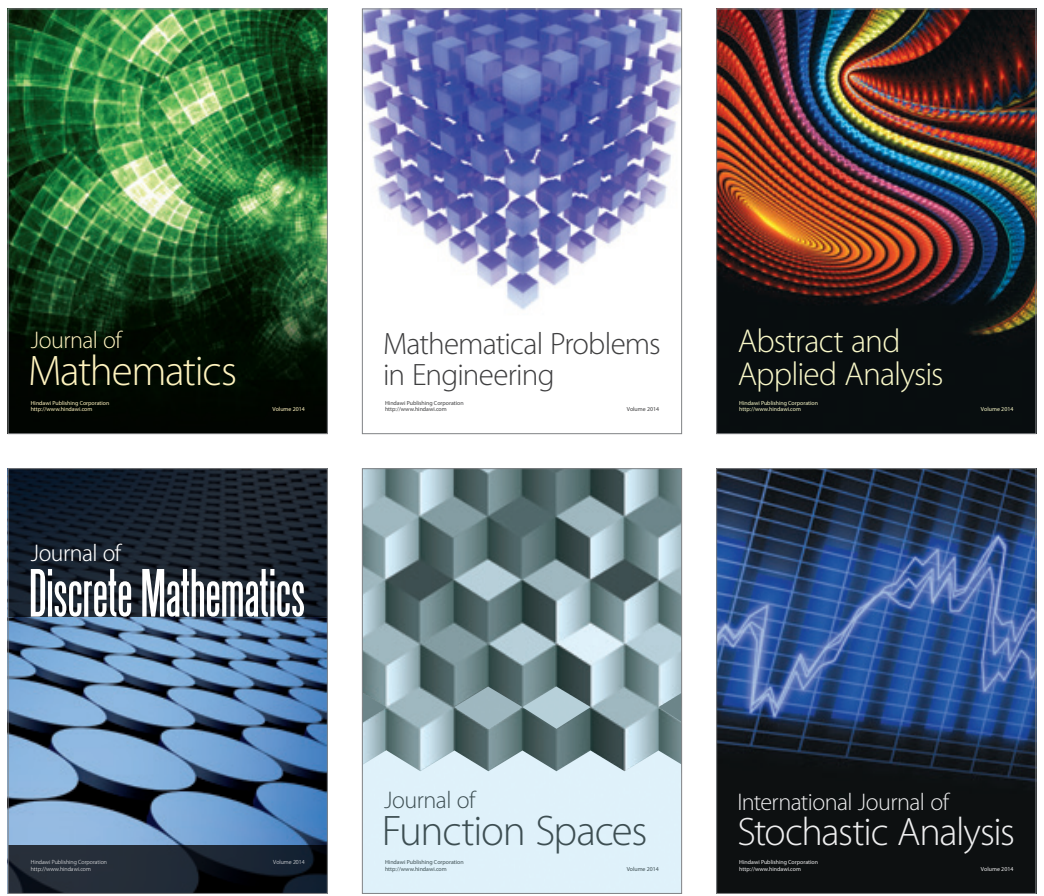

Journal of

Function Spaces

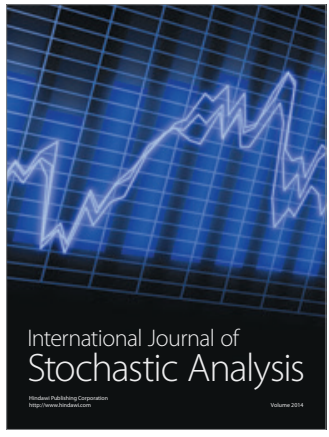

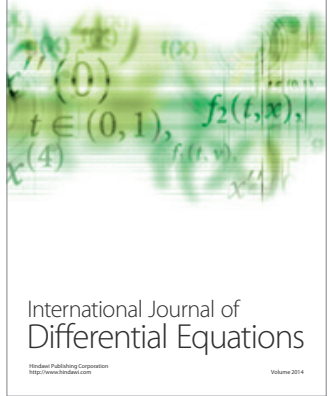
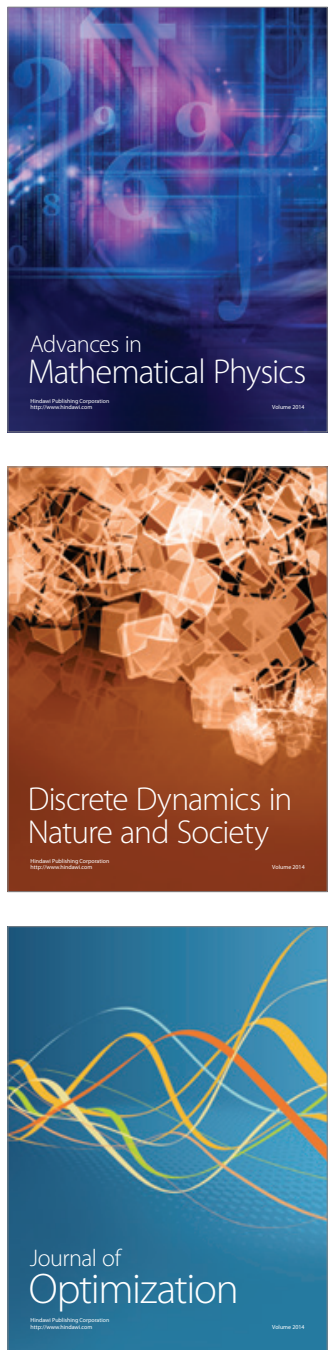